\title{
A study on the causes of operative failures after microwave ablation for primary hyperparathyroidism
}

\author{
Wei Ying $^{1} \cdot$ Zhao Zhen-long $^{1} \cdot$ Cao Xiao-jing ${ }^{1} \cdot$ Peng Li-li $^{1} \cdot$ Li Yan $^{1} \cdot$ Yu Ming-an ${ }^{1}$
}

Received: 10 August 2020 / Revised: 19 January 2021 / Accepted: 5 February 2021 / Published online: 2 March 2021

(C) The Author(s) 2021

\begin{abstract}
Objective To summarize the occurrence of operative failures after microwave ablation (MWA) in patients with primary hyperparathyroidism (pHPT), analyze the possible reasons, and explore strategies for preventing and managing these situations.

Methods This retrospective study reviewed 91 pHPT patients who underwent MWA from April 2015 to November 2019. A cure was defined as the reestablishment of normal calcium homeostasis lasting a minimum of 6 months. An operative failure was defined as a failure to normalize serum intact parathyroid hormone (iPTH) and/or calcium levels at 6 months or longer. Patients who encountered operative failures were compared with patients who were successfully cured.

Results Eighty-eight pHPT patients, consisting of 29 men and 59 women, were finally enrolled. The median follow-up duration was 15.9 months (IQR, 6.1-31.5 months). Seventy-eight patients (78/88, 88.6\%) were cured. Ten $(10 / 88,11.4 \%)$ patients experienced operative failure, including 9 persistent pHPT $(10.2 \%)$ and $1(1.1 \%)$ recurrent pHPT. Small parathyroid nodules (maximum diameter $<0.6 \mathrm{~cm}$ ) and incomplete ablation were the two key factors leading to operative failure. Of the 9 patients with a maximum nodule diameter less than $0.6 \mathrm{~cm}, 77.8 \%$ (7/9) of them encountered operative failure.

Conclusion Operative failure occurred in $11.4 \%$ of the pHPT patients who underwent MWA. The possibility of operative failure was increased when the maximum diameter of parathyroid nodule was less than $0.6 \mathrm{~cm}$. Complete ablation could help avoid operative failure.

Key Points

- Failed to ablate the target lesion and incomplete ablation were the key factors attributed to operative failures.

- When the maximum diameter of the parathyroid nodules is less than $0.6 \mathrm{~cm}$, the possibility of operative failure was higher.
\end{abstract}

Keywords Microwave radiation $\cdot$ Primary hyperparathyroidism $\cdot$ Parathyroid Hormone $\cdot$ Recurrence

\section{Abbreviations}

CEUS Contrast-enhanced ultrasound

MWA Microwave ablation

pHPT Primary hyperparathyroidism

PTH Parathyroid hormone

US Ultrasound

Yu Ming-an

yma301@163.com

1 Department of Interventional Medicine, China-Japan Friendship Hospital, No. 2 Ying-hua-yuan street, Chao-yang District, Beijing 100029, China

\section{Introduction}

Primary hyperparathyroidism (pHPT) is a common endocrine disorder, with a prevalence of $0.1-0.4 \%$ [1]. It is generally characterized by hypercalcemia and elevated parathyroid hormone (PTH) levels [2]. Nearly $20 \%$ of pHPT patients are asymptomatic when discovered; however, the disease has the potential to become symptomatic. The common clinical manifestations of pHPT include bone loss, kidney stones, and neurocognitive impairment [1-4].

Surgical resection is recommended as the definitive curative treatment for pHPT [5-7]. In recent years, after more than a decade of clinical application, ultrasound-guided thermal ablation has been demonstrated to be effective in inactivating parathyroid nodules and normalizing serum PTH and calcium 
levels [8-12]. A recent prospective study showed that microwave ablation (MWA) and surgical resection provided comparable results in terms of cure rate in the treatment of $\mathrm{pHPT}$ [10].

Despite these high cure rates, approximately $11-18 \%$ of patients did not achieve reestablished normal serum PTH and/ or calcium homeostasis after MWA $[10,11]$. It was reported that the most common causes of initial operative failure after surgical resection include unrecognized gland hyperplasia, double adenomas, ectopic location of the hyperfunctioning parathyroid gland, or operations performed by inexperienced or low-volume parathyroid surgeons [13-15]. However, currently, operative failures after MWA have not been systematically summarized and analyzed. Therefore, the purpose of this study was to summarize the occurrence of operative failures after MWA for pHPT, analyze the possible reasons, and explore strategies for preventing and managing these situations.

\section{Materials and methods}

\section{Study design}

This retrospective study protocol was approved by the Human Ethics Review Committee of the China-Japan Friendship Hospital. Written informed consent was obtained from each patient before the ablation procedure; informed consent was waived for this retrospective investigation. Between April 2015 and November 2019, the data of 91 pHPT patients (30 male and 61 female) treated with MWA were reviewed.

The inclusion criteria were as follows: (1) patients with symptomatic pHPT; (2) asymptomatic pHPT patients with one of the following conditions-(a) serum calcium level higher than the normal range; (b) T-score $<-2.5$ at the lumbar spine, total hip, femoral neck, or distal one-third of the radius, significant reduction in bone mineral density, and/or increased risk of a fragility fracture; (c) age $<50$ years; (3) patients with renal involvement-(a) creatinine clearance less than $60 \mathrm{ml} /$ min, (b) kidney stone or nephrocalcinosis found by ultrasound (US), CT, or abdominal radiography; (4) pHPT patients who were not eligible for surgery or refused surgery; and (5) pHPT patients unwilling to comply with observation protocols.

The exclusion criteria were as follows: (1) patients with severely abnormal coagulation function tests, such as prothrombin time $>18 \mathrm{~s}$, prothrombin activity $<60 \%$, or platelet count $<60 \times 10^{9} / \mathrm{L}$; (2) patients with underlying disease, such as cardiac insufficiency or hypertension, refractory to management with medication; and (3) patients with suspected parathyroid carcinoma based on imaging findings and laboratory tests (e.g., enlarged hypoechoic parathyroid glands with unclear margins; lymph node metastasis, markedly elevated iPTH levels and severe hypercalcemia). The flowchart of patient selection is shown in Fig. 1.
According to the guidelines, preoperative parathyroid fineneedle aspiration biopsy is not recommended due to undesirable consequences such as bleeding and needle track implantation $[7,16,17]$. In the present study, the parathyroid nodules of all patients were prelocalized with technetium 99-m-labeled sestamibi single-photon emission computed tomography (99mTc-sestamibi SPECT), routine US, and contrastenhanced ultrasound (CEUS). Clinical data, biochemical tests, US, CEUS, and 99mTc-sestamibi SPECT were used together for preoperative localization and qualitative diagnosis.

Before MWA, the diagnosis of pHPT on US and CEUS was based on the following criteria: (1) enlarged hypoechoic parathyroid glands with clearly defined margins; (2) no suspicion of lymph node metastasis; and (3) enlarged hypoechoic parathyroid glands showing hyperenhancement in artery phase on CEUS [7, 16, 17].

Information of each patient was obtained, including demographics, clinical parameters, laboratory indices, parathyroid lesion characteristics, and treatment variables (e.g., ablation time, complications, and pre- and post-MWA serum intact PTH [iPTH], calcium, phosphate, alkaline phosphatase [ALP], and 25-OH vitamin D levels). Patients who encountered operative failures were screened out and compared with patients who were successfully cured after MWA. The potential factors associated with operative failures were further analyzed.

\section{Microwave equipment and ablation technique}

The microwave platform (Intelligent Basic Type Microwave Tumor Ablation System, Nanjing ECO Microwave System) contains a microwave generator, a flexible coaxial cable, and a cooled-shaft antenna. The antenna is an internally cooled ablation needle (17-gauge, $10 \mathrm{~cm}$ in length, and a 3-mm active tip) covered with polytetrafluoroethylene to prevent adhesion. A LOGIQ E9 US system (GE Healthcare) equipped with a 6-15 MHz linear array transducer was used for US guidance and CEUS.

The patients were placed in a supine position with the neck extended. After the neck was sterilized, 40-60 mL normal saline (NS) was first injected into the area around the parathyroid nodule through an 18-G needle (Hakko Medical Co., Ltd.) to provide hydrodissection. Then, a lidocaine and NS mixture (1:3) was injected near the periparathyroid capsule for local anesthesia. The cooled MWA antenna was inserted freehand into the parathyroid gland under US guidance. During the ablation process, a continuous slow injection of NS was performed by an assistant to provide thermal insulation. A multipoint ablation strategy was adopted $[11,18]$. The power was $30 \mathrm{~W}$, and the radiation time was $15-25 \mathrm{~s}$ at each ablation point. The therapy was terminated when the hyperechoic zone covered the entire nodule. CEUS (SonoVue; Bracco) was performed 3-5 min later to assess the ablation efficacy. If the ablated nodule was covered by a nonenhanced zone, the ablation was considered complete. If 


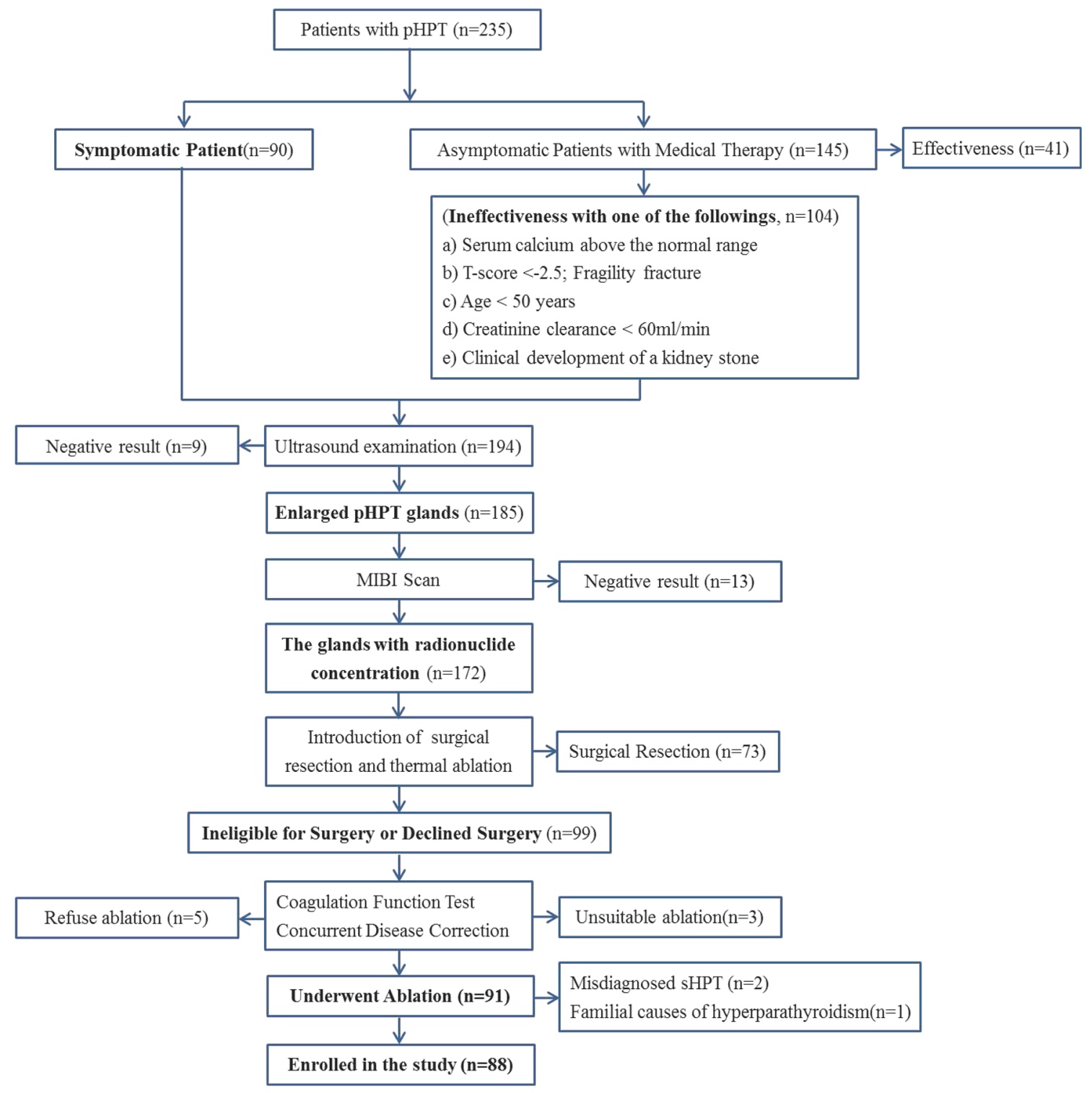

Fig. 1 The flowchart of patient selection. pHPT = primary hyperparathyroidism

there was nodular enhancement inside the nodule, further ablation was performed immediately. For bilateral nodule ablation, if there were no voice changes and no abnormal vocal cord movements on US after one side was ablated, then MWA would immediately be performed on the contralateral side. If there were any signs of RLN injury, the ablation was stopped, and the second session was suspended until RLN function recovered. At the end of the procedure, the puncture site was compressed for $30 \mathrm{~min}$, and the patient remained under observation for $2 \mathrm{~h}$ to monitor for potential complications.

\section{Follow-up}

Follow-up included routine US examination and blood biochemistry. The follow-up times were $2 \mathrm{~h}, 1$ day, 7 days, 1 month, and 3 months after the ablation and every 6 months thereafter. CEUS was only performed if the serum iPTH or calcium levels were elevated or if routine US showed suspicious nodules in the parathyroid region.

\section{Definition of a cure and operative failure}

Technical success was defined as a complete ablation after appropriate treatment according to the protocol. A cure was defined as the reestablishment of normal calcium homeostasis lasting a minimum of 6 months. An operative failure was defined as a failure to normalize serum iPTH and/or calcium levels at 6 months or longer after MWA. Persistent pHPT was defined as a failure to achieve normocalcemia within 6 months. Recurrent pHPT was defined as the recurrence of hypercalcemia and/or elevated iPTH level 6 months after MWA [7, 19]. 


\section{Statistical analysis}

Statistical analysis was performed using SPSS (version 20.0 for Windows; IBM) and Stata version 15.0 (StataCorp LLC). Continuous data are presented as the mean \pm standard deviation, and the median and $25-75 \%$ interquartile range (IQR) are used if the data did not fit a normal distribution. Categorical variables were analyzed with the chi-square test, and continuous variables were analyzed with the independent $t$-test or Mann-Whitney $U$ test. Differences were considered significant when $p<0.05$.

\section{Results}

The baseline and treatment parameters are summarized in Table 1 . Of the 91 patients reviewed, 2 secondary hyperparathyroidism patients who were misdiagnosed and 1 patient with familial cause of hyperparathyroidism were excluded. Finally, 88 sporadic pHPT patients, consisting of 29 men and 59 women, with 100 parathyroid glands (a

Table 1 Clinical and treatment characteristics of patients with PHPT

\begin{tabular}{ll}
\hline Characteristic & Data \\
\hline Number of cases & 88 \\
Gender (male/female) & $29 / 59$ \\
Mean age (years) & $56.5 \pm 16.8(18-85)$ \\
Symptomatic & 40 \\
Nephrolithiasis & 16 \\
Ostealgia & 16 \\
Fatigue & 14 \\
Neurocognitive impairment & 2 \\
Asymptomatic & 48 \\
25-Hydroxyvitamin D (nmol/L) & $31.8(14.0-81.2)$ \\
Pre-MWA iPTH (pg/ml) & $143.1(84.6-563.7)$ \\
Pre-MWA calcium (mg/dl) & $2.72 \pm 0.25(2.20-3.37)$ \\
ALP (U/L) & $78(51-180)$ \\
Nodules & 100 \\
Normal location & 99 \\
Upper pole & 28 \\
Lower pole & 71 \\
Ectopic location & 1 \\
Volume (cm $\left.{ }^{3}\right)$ & $0.504(0.038-6.804)$ \\
Maximum diameter (cm) & $1.3(0.5-3.4)$ \\
Ablation time (s) & $154(72-368)$ \\
Complication & 15 \\
Hoarseness & 4 \\
Bleeding & 3 \\
Transient hypocalcemia & 8 \\
\hline &
\end{tabular}

single gland in 77 patients, two glands in 10 patients, and three glands in 1 patient) were enrolled. The ages ranged from 18 to 85 years (mean, $56.5 \pm 16.8$ years). The median maximum diameter of the glands was $1.3 \mathrm{~cm}$ (IQR, $0.5-3.4 \mathrm{~cm})$.

\section{MWA procedure}

Complete ablation was achieved in one session in 87 cases and in two sessions in one case. The technical success rate was $100 \%$. The median ablation time was $154 \mathrm{~s}$ per nodule (IQR, $72-368 \mathrm{~s}$ ). The median follow-up duration was 15.9 months (IQR, 6.1-31.5 months). Of the entire cohort, 78 patients had normal levels of serum iPTH and calcium in the follow-up period after MWA, with a cure rate of $88.6 \%$ (78/88). The remaining $10(10 / 88,11.4 \%)$ patients experienced operative failure. Nine $(9 / 88,10.2 \%)$ patients had persistent $\mathrm{pHPT}$, and $1(1 / 88,1.1 \%)$ had recurrent pHPT. The time to recurrence was 9 months after MWA.

\section{Cause, management, and outcome of patients with operative failure}

Of the 10 patients who experienced operative failure, 3 showed increased levels of serum iPTH and calcium, and the other 7 only showed an increase in the serum iPTH level while maintaining a serum calcium level (Table 2).

In five of the 10 cases of operative failure, the ablation zone covered the soft tissue close to the pHPT nodule but not the pHPT nodule itself. The maximum diameter of these nodules ranged from 0.4 to $0.5 \mathrm{~cm}$, and the volume ranged from 0.024 to $0.047 \mathrm{~cm}^{3}$. For the other two patients with Hashimoto's thyroiditis, the adjacent lymph nodes rather than the pHPT nodules were ablated. The lymph nodes were near the parathyroid nodules (maximum diameter, $0.9-1.0 \mathrm{~cm}$; volume, $0.196-0.203 \mathrm{~cm}^{3}$ ). In the other 3 cases, incomplete ablation was found by elevated serum iPTH levels 1-3 months after MWA. The residual lesions showed hyperenhancement on CEUS (Fig. 2).

Of these 10 patients, three with hypercalcemia underwent successful surgical resection (2 patients) or a second ablation (1 patient), respectively. Three patients with residual lesions received additional ablation and were cured. The other 4 patients with very small nodules and only elevated serum iPTH levels received vitamin $\mathrm{D}$, calcitriol supplementation, and regular follow-up. The causes, management, and outcomes of patients with operative failures after MWA are outlined in Table 3.

\section{Risk factors for operative failure}

There were significant differences in the pre-MWA iPTH level $(p=0.016)$, serum calcium level $(p=0.03)$, lesion diameter $(p=0.000)$, and lesion volume $(p=0.000)$ between the cured 
Table 2 The cases of operative failure after MWA

\begin{tabular}{|c|c|c|c|c|c|c|c|c|c|c|c|c|}
\hline \multirow[t]{2}{*}{ Case } & \multirow[t]{2}{*}{ Sex } & \multirow{2}{*}{$\begin{array}{l}\text { Age } \\
\text { (year) }\end{array}$} & \multirow{2}{*}{$\begin{array}{l}\text { Nodule } \\
\text { diameter }(\mathrm{cm})\end{array}$} & \multicolumn{3}{|c|}{ Pre-MWA } & \multicolumn{2}{|c|}{ 1D post-MWA } & \multicolumn{4}{|c|}{ Persistent/recurrent PHPT } \\
\hline & & & & $\begin{array}{l}\text { iPTH } \\
(\mathrm{pg} / \mathrm{mL})\end{array}$ & $\begin{array}{l}\text { Calcium } \\
(\mathrm{mmol} / \mathrm{L})\end{array}$ & $\begin{array}{l}\text { Vitamin D } \\
(\mathrm{nmol} / \mathrm{L})\end{array}$ & $\begin{array}{l}\text { iPTH } \\
(\mathrm{pg} / \mathrm{mL})\end{array}$ & $\begin{array}{l}\text { Calcium } \\
(\mathrm{mmol} / \mathrm{L})\end{array}$ & $\begin{array}{l}\text { Time of } \\
\text { detection }\end{array}$ & $\begin{array}{l}\text { iPTH } \\
(\mathrm{pg} / \mathrm{mL})\end{array}$ & $\begin{array}{l}\text { Calcium } \\
(\mathrm{mmol} / \mathrm{L})\end{array}$ & $\begin{array}{l}\text { Vitamin D } \\
(\mathrm{nmol} / \mathrm{L})\end{array}$ \\
\hline 1 & $\mathrm{~F}$ & 73 & 1.2 & 139.6 & 2.45 & 42.1 & 31.8 & 1.96 & $3 \mathrm{M}$ & 121.9 & 2.56 & 59.6 \\
\hline 2 & $\mathrm{~F}$ & 73 & 1.6 & 352.0 & 2.55 & 24.3 & 12 & 2.48 & $1 \mathrm{M}$ & 273.1 & 2.18 & 20.8 \\
\hline 3 & $\mathrm{M}$ & 75 & 2.2 & 230.5 & 3.01 & 37.1 & 15.3 & 2.82 & $1 \mathrm{M}$ & 88.6 & 2.86 & 46.7 \\
\hline 4 & $\mathrm{M}$ & 36 & 0.5 & 118.8 & 2.88 & 42.1 & 92.2 & 2.88 & $1 \mathrm{D}$ & 92.2 & 2.88 & - \\
\hline 5 & $\mathrm{~F}$ & 42 & 0.4 & 121.8 & 2.31 & 30.7 & 60.7 & 2.14 & $15 \mathrm{M}$ & 120.1 & 2.28 & 29.4 \\
\hline 6 & $\mathrm{~F}$ & 61 & 0.5 & 103.4 & 2.43 & 70.1 & 96.9 & 2.51 & $1 \mathrm{D}$ & 96.9 & 2.51 & - \\
\hline 7 & $\mathrm{M}$ & 40 & 1 & 89.5 & 2.2 & 33 & 119.7 & 2.19 & $1 \mathrm{D}$ & 119.7 & 2.19 & - \\
\hline 8 & $\mathrm{M}$ & 58 & 0.4 & 81.8 & 2.99 & 46.2 & 99.7 & 2.83 & $1 \mathrm{D}$ & 99.7 & 2.83 & - \\
\hline 9 & $\mathrm{M}$ & 35 & 0.9 & 128.1 & 2.36 & 54 & 98.5 & 2.39 & $1 \mathrm{D}$ & 98.5 & 2.39 & - \\
\hline 10 & M & 37 & 0.5 & 109.4 & 2.54 & 37.9 & 106.3 & 2.43 & $1 \mathrm{D}$ & 106.3 & 2.43 & - \\
\hline
\end{tabular}

patients and the patients with operative failure due to missed or false punctures (Table 4). Serum calcium $(r=0.241, p=$ $0.027)$ and $\mathrm{iPTH}(r=0.560, p=0.000)$ values were positively correlated with the maximum diameter of the parathyroid nodule. According to the ROC analysis, a maximum diameter less than $0.6 \mathrm{~cm}$ or a volume smaller than $0.208 \mathrm{~cm}^{3}$ resulted in the

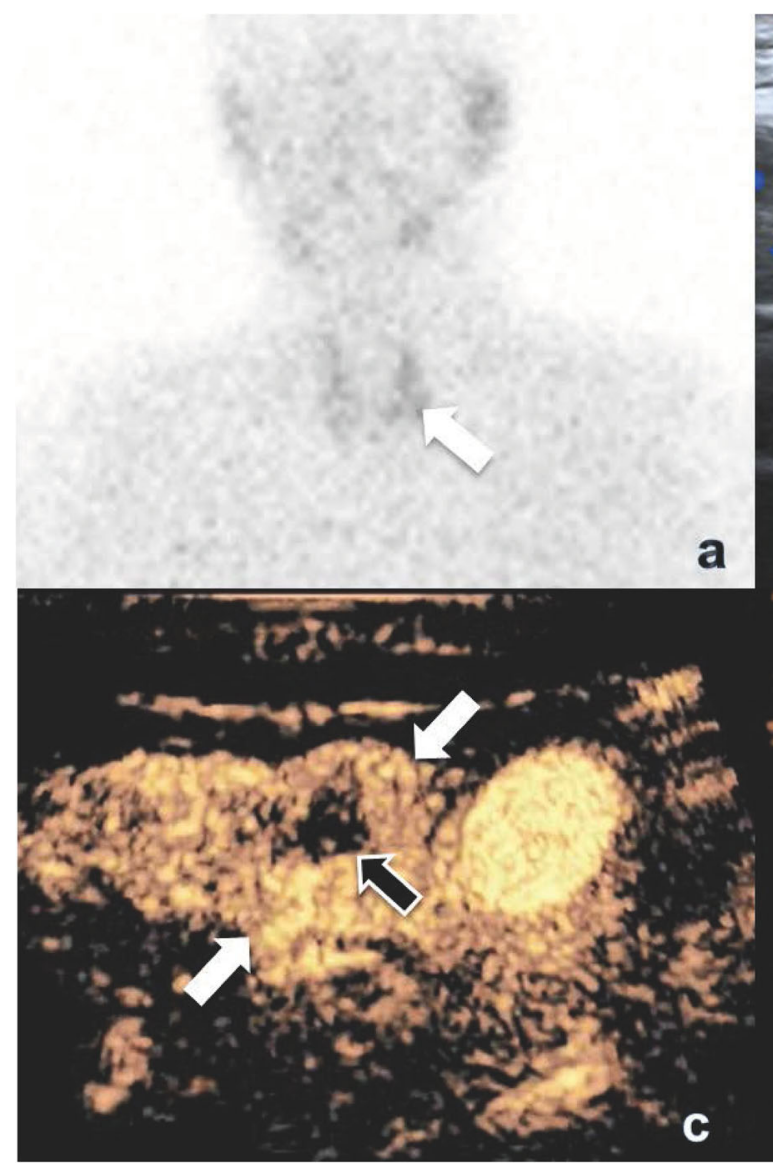

Fig. 2 CEUS shows a hyperenhanced residual lesion in a 75-year male patient with pHPT. a Three months after the first MWA, there was radioactive concentration in residual lesion (arrow) on MIBI scan. b Color Doppler showed abundant blood flow signals around the hypoechoic ablation zone (arrows). c There was active area-annular

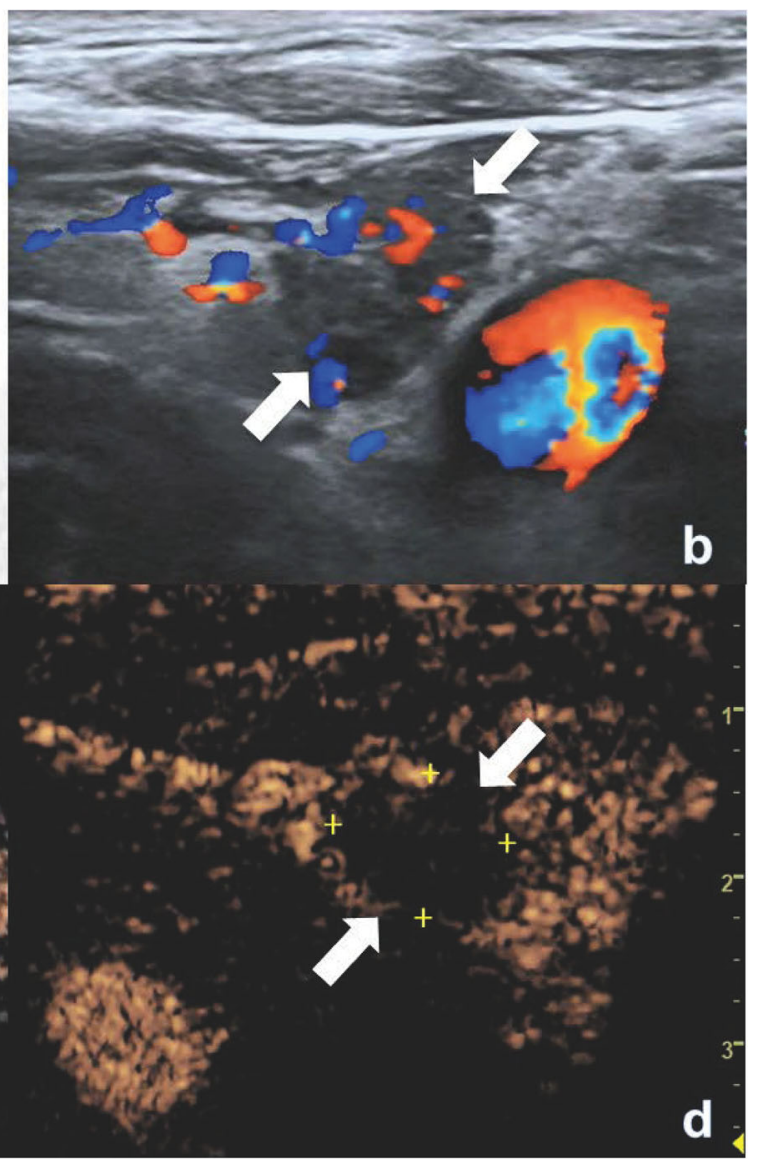

hyperenhancement area-(white arrows) around ablation zone (black arrow) on CEUS. d After additional ablation, non-enhancement (arrows) was shown on CEUS. pHPT, primary hyperparathyroidism; CEUS, contrasted-enhanced ultrasound; MWA, microwave ablation; MIBI, technetium $99 \mathrm{~m}\left({ }^{99 \mathrm{~m}} \mathrm{Tc}\right)$ sestamibi 
Table 3 The causes of operative failure after MWA

\begin{tabular}{llll}
\hline Cause of operative failure & $\begin{array}{l}\text { Number of case } \\
(n)\end{array}$ & Management & Outcome \\
\hline Failed to ablate target lesion & 7 & - & - \\
- Too small nodules (diameter $\leq$ & 5 & $\begin{array}{l}\text { Surgery (1), ablation (1), medical } \\
\text { treatment }(3)\end{array}$ & Cure \\
$0.5 \mathrm{~cm})$ & 2 & Surgery (1), medical treatment (1) & Cure \\
- Confused with lymph nodes & 3 & Second ablation & Cure \\
Incomplete ablation & 3 & &
\end{tabular}

highest Youden index values for operative failure. Of the nine patients with lesions with a maximum diameter less than 0.6 $\mathrm{cm}, 77.8 \%$ (7/9) of the patients encountered operative failure.

In the three patients that underwent incomplete ablation, the univariate analysis results showed that there were no parameters associated with operative failure.

\section{Complications}

As a major complication, voice change occurred in 4 patients (4.5\%), but all improved within 2-3 months. As a minor complication, hematoma occurred in $3(3.4 \%)$ patients, and all hematoma was treated successfully with pressure and thrombin injections (Hemocoagulase Bothrops Atrox for Injection, Nuokang Pharmaceutical Company). Mild postoperative transient hypocalcemia accompanied by perioral and limb numbness occurred in 8 patients $(9.1 \%)$. Their symptoms were gradually relieved by the appropriate use of calcitriol and supplemental calcium.

\section{Discussion}

In recent years, US-guided thermal ablation for pHPT has been demonstrated to be a promising option because of its minimal invasiveness, safety, promising efficacy, wide indications, and rapid postoperative recovery [8-12]. However, the cure rate of MWA is approximately $82.1-89.4 \%$, and still there are some patients have failed procedures [9-11]. Therefore, it is essential to summarize the cases of operative failure after MWA, analyze the possible reasons to prevent these situations, and increase the cure rate.

This retrospective study reviewed 88 patients who underwent MWA for sporadic pHPT. Up to now, the present study included the largest number of pHPT patients who have undergone MWA $[9,10,12]$. The results demonstrated operative failure in $11.4 \%$ of patients. According to the statistical results, when the maximum diameter of the parathyroid nodules was less than $0.6 \mathrm{~cm}$, the possibility of operative failure was obviously higher, at $77.8 \%$. According to our experience, during hydrodissection, the liquid can diffuse unevenly and cause nodular changes in the surrounding tissues. As a result, the echogenicity of the liquid was similar to that of the parathyroid nodules - both were hypoechoic, which easily misguided the operator and led to false targets (Fig. 3). In fact, some small parathyroid nodules might be missed even during open surgery [14]. In cases of missed puncture, even if the thermal ablation zone was close to the nodule, it was difficult for the heat energy to reach the pHPT nodule due to the barrier
Table 4 Comparison of relevant clinical parameters between the cured cases and the cases of operative failure due to missing and false puncture

\begin{tabular}{lccc}
\hline Variables & Operative failure $(7)$ & Cure $(77)$ & $p$ \\
\hline Female $(n)$ & 3 & 53 & 0.215 \\
Age $($ years $)$ & $44.1 \pm 10.8$ & $56.9 \pm 17.0$ & 0.055 \\
Pre-MWA iPTH (pg/ml) & $109.4(81.8-121.8)$ & $151.8(86.3-579.4)$ & 0.016 \\
Serum calcium (mmol/L) & $2.53 \pm 0.30$ & $2.74 \pm 0.24$ & 0.030 \\
Serum phosphorus (mmol/L) & $0.95 \pm 0.18$ & $0.84 \pm 0.19$ & 0.160 \\
ALP (U/L) & $68(54-85)$ & $78(50-195)$ & 0.124 \\
Regenerative growth & & & \\
25(OH)D3(nmol/L) & $42.1(30.7-54)$ & $32.8(13-83.5)$ & 0.054 \\
GFR (mL/min) & $95.9(83.1-114.2)$ & $97.6(41.4-128.5)$ & 0.492 \\
CCR (umol/L) & $77(45.5-91.8)$ & $60.1(43.1-108.3)$ & 0.345 \\
Ur (mmol/L) & $4.5(2.2-7.4)$ & $4.3(3.3-5.9)$ & 0.654 \\
Max diameter (cm) & $0.5(0.4-0.9)$ & $1.3(0.6-3.6)$ & $<0.001$ \\
Volume (ml) & $0.042(0.024-0.196)$ & $0.580(0.074-8.709)$ & $<0.001$ \\
\hline
\end{tabular}

$i P T H$, intact parathyroid hormone; $A L P$, alkaline phosphatase; $M W A$, microwave ablation; $G F R$, glomerular filtration rate; $C C R$, creatinine clearance rate; $U r$, urea 


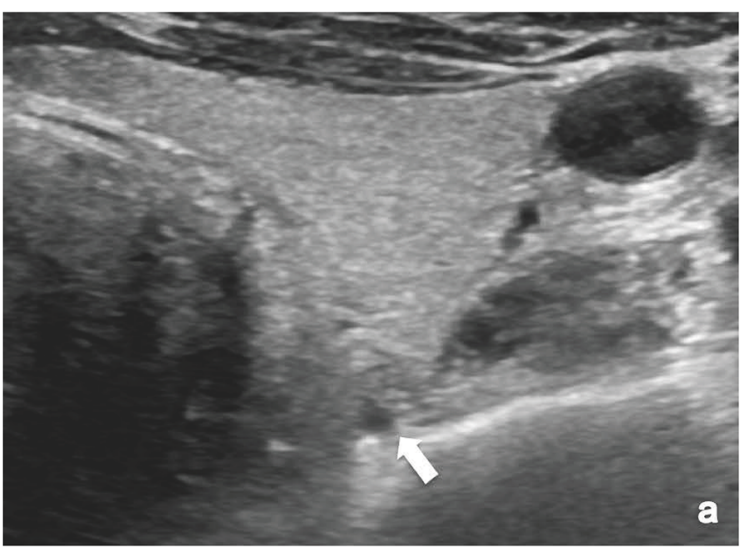

Fig. 3 Nodular changes in the surrounding tissues during hydrodissection. a Routine US showed a hypoechoic pHPT nodule with maximum diameter of $0.4 \mathrm{~cm}$ in a 58 -year male patient. b During hydrodissection, the liquid (yellow arrow) diffuse unevenly and cause

of the nodule capsule and the heat sink caused by the isolation fluid, thereby causing incomplete ablation. Therefore, several strategies could be employed to prevent false puncture: (i) before injecting the isolation solution, the PTC needle could be inserted into the nodule to anchor the nodule; (ii) the ablation antenna could be directly inserted into the nodule first, before establishing hydrodissection. In other words, prelocalization should be adopted to avoid losing the target nodules and prevent operative failure for patients with small lesions.

For patients with lymphocytic thyroiditis, the perithyroidal central compartment lymph nodes can commonly be misdiagnosed as parathyroid nodules [19]. Some parathyroid nodules were similar to the lymph nodes in size and echogenicity, making it difficult to distinguish between the two and sometimes resulting in false puncture during ablation. In these cases, CEUS could be helpful. According to our experience, the lymph nodes in patients with Hashimoto's thyroiditis were usually hypoenhanced in the arterial phase on

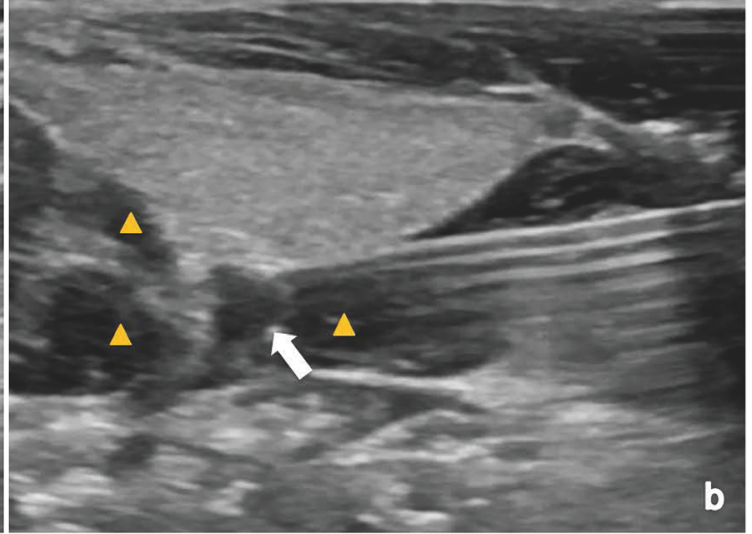

nodular changes in the surrounding tissues. Both the liquid and the pHPT nodule (arrow) were hypoechoic. pHPT, primary hyperparathyroidism

CEUS, while the pHPT nodules due to hypermetabolism were generally hyperenhanced.

Partial remission of hyperparathyroidism caused by incomplete ablation had also been reported in previous studies [10, 20]. Based on the three patients with operative failure due to incomplete ablation in the present study, our recommendations are summarized as follows: (i) a complete ablation should contain both hypoechoic hyperplastic nodules and normal parathyroid tissue, which often appear hyperechoic on US, especially after the injection of the isolation solution (Fig. 4); and (ii) intraoperative iPTH monitoring is one of many adjuncts to achieve complete ablation according to the surgical procedure [21].

Transient hoarseness caused by impaired mobility of the ipsilateral vocal cord was reported in previous studies on thermal ablation of pHPT. The rate was varying from 6 to $38 \%$ $[11,12,22]$. In the present study, hoarseness occurred in 4 patients $(4.5 \%)$, but all patients' voice improved within 2-3 months. The rate was higher than that reported in the thermal

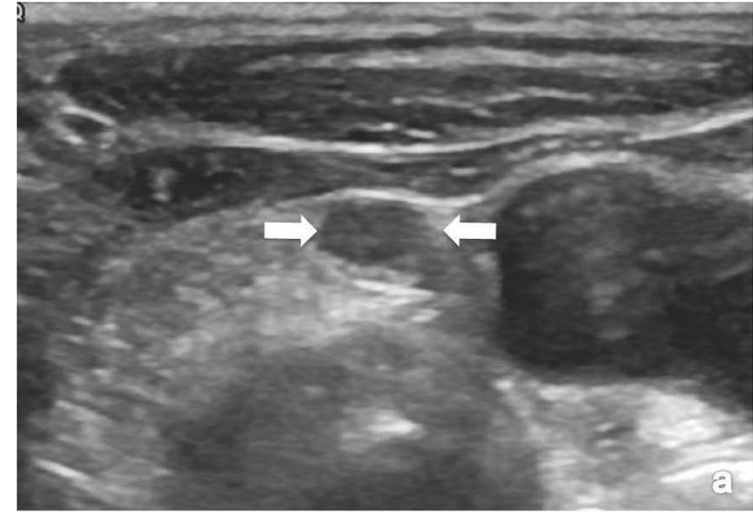

Fig. 4 The hypoechoic hyperplastic parathyroid nodule and hyperechoic normal parathyroid tissue could be clearly visualized after establishment of hydrodissection. a Routine US showed a hypoechoic pHPT nodule (white arrows) in a 45-year female patients. b After the injection of

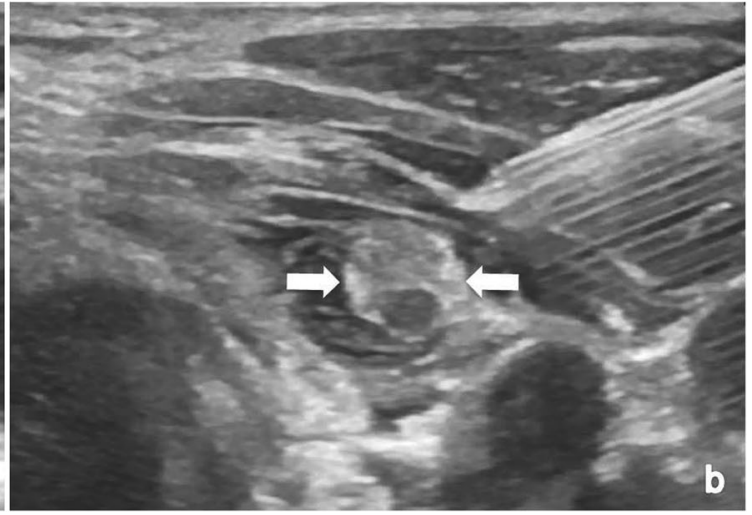

isolation solution, the hypoechoic hyperplasic parathyroid nodule (white arrows) and hyperechoic normal parathyroid tissue were clearly displayed on US. pHPT, primary hyperparathyroidism; US, ultrasound 
ablation of thyroid nodules $(1.5 \%)$ and in parathyroidectomies $(2.4 \%)[23,24]$. The reason might be that some parathyroid nodules were too close to the tracheoesophageal groove, where the recurrent laryngeal nerve (RLN) was located; and the nerves were very sensitive to thermal stimulation. Therefore, minimizing heat exposure to the RLN is extremely critical during ablation. There are several strategies that could be employed to protect the nerve. First, effective hydrodissection technology can effectively reduce thermal damage to the RLN. Second, accurate puncture and ablation monitored by US could help protect RLN against thermal injury. Third, short time and repeated radiation with low power is helpful in preventing thermal injuries to the surrounding critical structures.

There are still a few limitations in the present study. First, no pathological results were obtained because biopsy was not recommended according to the guidelines. The number of operative failures was relatively small, and future studies with large sample sizes might help to provide more definitive results. Third, this is a retrospective study, and there may be selection bias. Prospective studies are needed.

In conclusion, MWA is an effective treatment method. However, operative failure is still inevitable in $11.4 \%$ patients. The small size (maximum diameter $<0.6 \mathrm{~cm}$ ) of pHPT nodule was one of the key factors leading to operative failure. Prelocalization and mastery of the details of the ablation technique could help avoid operative failure and improve the cure rate by increasing the rate of complete ablation.

Acknowledgements Authors' roles: study design - Wei Ying and Yu Ming-an. Study conduct: Yu Ming-an. Data collection: Wei Ying, Peng Li-li, Li Yan, and Cao Xiao-jing. Data analysis: Wei Ying and Zhao Zhen-long. Data interpretation: Wei Ying. Drafting of manuscript: Wei Ying. Revising manuscript content: Wei Ying, Yu Ming-an. Approving final version of manuscript: Wei Ying, Zhao Zhen-long, Cao Xiao-jing, Peng Li-li, Li Yan, and Yu Ming-an.

Funding This study has received funding from the Beijing Municipal Science \& Technology Commission(Z181100001718135) and the Beijing University of Chemical Technology-China-Japan Friendship Hospital Biomedical Transformation Joint Fund Project (PYBZ1804).

\section{Declarations}

Guarantor The scientific guarantor of this publication is corresponding author Yu Ming-an.

Conflict of interest We confirm that the article is not under consideration for publication elsewhere, none of the parts of the article have been sent by mail, each author has participated sufficiently in any submission to take public responsibility for its content, publication is approved by all authors and tacitly or explicitly by the responsible authorities where the work was carried out, and all authors have no conflicts of interest including financial or personal relationships that inappropriately influence our actions of the work beginning submitted.
Statistics and biometry Data analysis has been finished by Ying Wei and no complex statistical methods were necessary for this paper.

Informed consent Written informed consent was obtained from all patients in this study.

Ethical approval The study protocol was approved by the Human Ethics Review Committee of the China-Japan Friendship Hospital (2015-GZR-77).

\section{Methodology}

- retrospective/observational study

Open Access This article is licensed under a Creative Commons Attribution 4.0 International License, which permits use, sharing, adaptation, distribution and reproduction in any medium or format, as long as you give appropriate credit to the original author(s) and the source, provide a link to the Creative Commons licence, and indicate if changes were made. The images or other third party material in this article are included in the article's Creative Commons licence, unless indicated otherwise in a credit line to the material. If material is not included in the article's Creative Commons licence and your intended use is not permitted by statutory regulation or exceeds the permitted use, you will need to obtain permission directly from the copyright holder. To view a copy of this licence, visit http://creativecommons.org/licenses/by/4.0/.

\section{References}

1. Bilezikian JP, Bandeira L, Khan A, Cusano NE (2018) Hyperparathyroidism. Lancet 391:168-178. https://doi.org/10. 1016/S0140-6736(17)31430-7

2. Applewhite MK, Schneider DF (2014) Mild primary hyperparathyroidism: a literature review. Oncologist 19:919-929. https://doi. org/10.1634/theoncologist.2014-0084

3. Minisola S, Gianotti L, Bhadada S, Silverberg SJ (2018) Classical complications of primary hyperparathyroidism. Best Pract Res Clin Endocrinol Metab 32:791-803. https://doi.org/10.1016/j.beem. 2018.09.001

4. Ejlsmark-Svensson H, Bislev LS, Lajlev S et al (2018) Prevalence and risk of vertebral fractures in primary hyperparathyroidism: a nested case-control study. J Bone Miner Res 33:1657-1664. https://doi.org/10.1002/jbmr.3461

5. Dy BM, Grant CS, Wermers RA et al (2012) Changes in bone mineral density after surgical intervention for primary hyperparathyroidism. Surgery 152:1051-1058. https://doi.org/10.1016/j. surg.2012.08.015

6. Silverberg SJ, Shane E, Jacobs TP, Siris E, Bilezikian JP (1999) A 10-year prospective study of primary hyperparathyroidism with or without parathyroid surgery. N Engl J Med 341:1249-1255. https:// doi.org/10.1056/NEJM199910213411701

7. Wilhelm SM, Wang TS, Ruan DT et al (2016) The American Association of Endocrine Surgeons Guidelines for Definitive Management of Primary Hyperparathyroidism. JAMA Surg 151: 959-968. https://doi.org/10.1001/jamasurg.2016.2310

8. Appelbaum L, Goldberg SN, Ierace T, Mauri G, Solbiati L (2020) US-guided laser treatment of parathyroid adenomas. Int $\mathrm{J}$ Hyperthermia 37:366-372. https://doi.org/10.1080/02656736. 2020.1750712

9. Fan BQ, He XW, Chen HH, Zhang WM, Tang W (2019) USguided microwave ablation for primary hyperparathyroidism: a safety and efficacy study. Eur Radiol 29:5607-5616. https://doi. org/10.1007/s00330-019-06078-y 
10. Liu F, Yu X, Liu Z et al (2019) Comparison of ultrasound-guided percutaneous microwave ablation and parathyroidectomy for primary hyperparathyroidism. Int J Hyperthermia 36:835-840. https:// doi.org/10.1080/02656736.2019.1645365

11. Wei Y, Peng L, Li Y, Zhao ZL, Yu MA (2020) Clinical study on safety and efficacy of microwave ablation for primary hyperparathyroidism. Korean J Radiol 21:572-581. https://doi.org/10.3348/ kjr.2019.0593

12. Ye J, Huang W, Huang G et al (2020) Efficacy and safety of USguided thermal ablation for primary hyperparathyroidism: a systematic review and meta-analysis. Int J Hyperthermia 37:245-253. https://doi.org/10.1080/02656736.2020.1734673

13. Chen H, Wang TS, Yen TW et al (2010) Operative failures after parathyroidectomy for hyperparathyroidism: the influence of surgical volume. Ann Surg 252:691-695. https://doi.org/10.1097/SLA. 0b013e3181f698df

14. Alhefdhi A, Schneider DF, Sippel R, Chen H (2014) Recurrent and persistence primary hyperparathyroidism occurs more frequently in patients with double adenomas. J Surg Res 190:198-202. https:// doi.org/10.1016/j.jss.2014.02.024

15. Mazotas IG, Yen T, Doffek K et al (2020) Persistent/recurrent primary hyperparathyroidism: does the number of abnormal glands play a role? J Surg Res 246:335-341. https://doi.org/10.1016/j.jss. 2019.08.007

16. Zini M, Attanasio R, Cesareo R et al (2012) AME position statement: primary hyperparathyroidism in clinical practice. $\mathrm{J}$ Endocrinol Invest 35:2-21

17. Bilezikian JP, Brandi ML, Eastell R et al (2014) Guidelines for the management of asymptomatic primary hyperparathyroidism: summary statement from the Fourth International Workshop. J Clin Endocrinol Metab 99:3561-3569. https://doi.org/10.1210/jc.2014-1413

18. Zhuo L, Peng LL, Zhang YM et al (2017) US-guided microwave ablation of hyperplastic parathyroid glands: safety and efficacy in patients with end-stage renal disease-a pilot study. Radiology 282: 576-584. https://doi.org/10.1148/radiol.2016151875

19. Johnson NA, Tublin ME, Ogilvie JB (2007) Parathyroid imaging: technique and role in the preoperative evaluation of primary hyperparathyroidism. AJR Am J Roentgenol 188:1706-1715. https://doi. org/10.2214/AJR.06.0938

20. Ha EJ, Baek JH, Baek SM (2020) Minimally invasive treatment for benign parathyroid lesions: treatment efficacy and safety based on nodule characteristics. Korean J Radiol. https://doi.org/10.3348/kjr. 2020.0037

21. Schneider DF, Mazeh H, Chen H, Sippel RS (2014) Predictors of recurrence in primary hyperparathyroidism: an analysis of 1386 cases. Ann Surg 259:563-568. https://doi.org/10.1097/SLA. 0000000000000207

22. Korkusuz H, Wolf T, Grunwald F (2018) Feasibility of bipolar radiofrequency ablation in patients with parathyroid adenoma: a first evaluation. Int J Hyperthermia 34:639-643. https://doi.org/ 10.1080/02656736.2018.1453552

23. Abdulla AG, Ituarte PH, Harari A, Wu JX, Yeh MW (2015) Trends in the frequency and quality of parathyroid surgery: analysis of 17 , 082 cases over 10 years. Ann Surg 261:746-750. https://doi.org/10. 1097/SLA.0000000000000812

24. Kim C, Lee JH, Choi YJ et al (2017) Complications encountered in ultrasonography-guided radiofrequency ablation of benign thyroid nodules and recurrent thyroid cancers. Eur Radiol 27:3128-3137. https://doi.org/10.1007/s00330-016-4690-y

Publisher's note Springer Nature remains neutral with regard to jurisdictional claims in published maps and institutional affiliations. 